\title{
Gestión de RR.PP. Internacionales: la aventura de Sentir Canario Gobcan.es
}

\author{
International Public Relations Management: Sentir Canario Gobcan.es
}

\author{
Araceli ÁLVAREZ DÍAZ \\ Universidad de La Laguna, Tenerife. (España) \\ aalvdiaw@gobiernodecanarias.org
}

Recibido: 13 de enero del 2019

Aceptado: 12 de febrero 2019

\begin{abstract}
Resumen
Este trabajo analiza los contenidos, las fuentes y la dinámica de funcionamiento de un proyecto de comunicación institucional que inició su andadura en el mes de julio de 2017 de la mano de la Dirección General de Emigración del Gobierno de Canarias con el objetivo de crear comunidad entre los emigrantes canarios y sus descendientes que residen fuera de Canarias. Dicho proyecto, denominado Sentir Canario Gobcan.es, que consta de una página de Facebook de actualización diaria en la que se inserta, además, un programa de radio semanal, persigue fomentar el vínculo de pertenencia de las comunidades de canarios que viven fuera de las islas. Se trata de un canal participativo en el que se da voz a dichos colectivos a través de la publicación de contenidos relacionados con las actividades que desarrollan, tanto de forma grupal como individualmente. Los objetivos del estudio son analizar, por un lado, los contenidos de esta red social, de actualización diaria, aunque con distintos ritmos los fines de semana; determinar cuáles son los criterios de selección de dichos contenidos así como las fuentes; y concretar cuál es el nivel de participación de estas entidades canarias en los contenidos publicados en la página.
\end{abstract}

\begin{abstract}
This work analyzes the contents, sources, and operating dynamics of an institutional communication project that began in July 2017 by the General Directorate of Emigration of the Government of the Canary Islands with the aim of creating community among Canarian emigrants and their descendants residing outside the Canary Islands. This project, called Sentir Canario Gobcan.es, which consists of a Facebook page updated daily in which a weekly radio program is also inserted, seeks to promote the link of belonging of the communities of canaries that live outside the islands. It is a participatory channel in which these groups are given a voice through the publication of content related to the activities they carry out, both as a group and individually. The objectives of the study are to analyze, on the one hand, the contents of this social network, updated daily, although at different rates on weekends; determine what are the selection criteria for said content as well as the sources; and specify what is the level of participation of these Canarian entities in the content published on the web.
\end{abstract}

Facultad de Ciencias Sociales y de la Comunicación

Universidad de La Laguna

Avenida César Manrique, s/n; Campus de Guajara

38071 La Laguna, Tenerife (Islas Canarias - España)

www.revistapangea.org | Pág. 1 
Palabras clave: Redes; Comunicación institucional; Comunidad; Emigración.

Keywords: Networks; Institutional comunication; Community; Emigration.

\section{Introducción}

Sentir Canario Gobcan.es es una página de Facebook anclada en los servidores y bajo el dominio del Gobierno de Canarias (gobiernodecanarias.org), cuyos contenidos están gestionados por la empresa Sales UP! mediante un contrato menor. Este proyecto depende de la Dirección General de Emigración del Gobierno de Canarias que, a su vez, está adscrita a la Viceconsejería de Acción Exterior. Se trata de una iniciativa de comunicación institucional destinada a los canarios emigrados y sus descendientes, que residen básicamente en América Latina y en el resto del territorio nacional, con el objetivo de crear comunidad y fomentar el sentimiento de pertenencia a las islas.

La legislación en esta materia en España cuenta, entre otras disposiciones, con la Ley 40/2006, de 14 de diciembre, del Estatuto de la ciudadanía española en el exterior; el Real Decreto 230/2008, de 15 de febrero, por el que se regula el Consejo general de la Ciudadanía española en el exterior; y el Real Decreto 1960/2009, de 18 de diciembre, por el que se regulan los Consejos de residentes españoles en el extranjero. Por su parte, en Canarias se dispone de la Ley 4/1986, de 25 de junio, de Entidades Canarias en el Exterior.

Según el artículo 5. 2. de la citada ley de ámbito regional, las entidades reconocidas por la Comunidad Autónoma tendrán derecho a participar en los medios de comunicación social de la comunidad autónoma y a recabar, canalizar y transmitir los proyectos de actividades que tengan su origen en la comunidad canaria de su ámbito.

Este proyecto dio sus primeros pasos el 3 de julio de 2017, y se ha ido consolidando en los dos años siguientes con la participación de la mayor parte de las entidades canarias que existen en América Latina así como en el territorio peninsular y, de forma residual, en el continente europeo.

El único precedente en España de páginas de este tipo, aunque con un funcionamiento distinto es el canal que tiene el Gobierno vasco en Facebook, denominado 'euskaletxeak.net, portal de y para los centros vascos', que se define como punto de encuentro de todas las comunidades vascas en el exterior, y que comenzó su andadura en noviembre de 2011. En este caso, se replican las publicaciones de las páginas de Facebook de los centros vascos y se les añade un pequeño texto o referencia. Además, el número de post cada día es variable y no hay un horario definido. 
Por su parte, la Generalitat catalana cuenta con una página de Facebook denominada 'Catalans exterior' dependiente de la Secretaria de Asuntos Exteriores. Se trata de una red social cuyos contenidos tienen como fuentes principales el portal exterior.cat que se define como "el portal que explica lo que hace Cataluña en el mundo" que gestiona el Grup Comunicaiò 21 y exteriorsgencat.cat, la web de la Generalitat de Cataluña.

Otras comunidades autónomas como Galicia, que cuenta con una importante comunidad de gallegos residentes fuera de España, tiene una Secretaría General de Emigración que dispone de un portal en Facebook, en este caso denominado 'GaliciaAberta', pero con una dinámica de funcionamiento distinta ya que se basa fundamentalmente en dar a conocer la actividad institucional de su titular así como de publicitar las medidas que pone en marcha la Xunta de Galicia destinadas a los emigrantes. Similar es el caso de Asturias que también dispone de una página web institucional denominada 'Emigrastur' destinada a contenidos vinculados con la emigración asturiana.

\section{Objetivos}

Los objetivos del estudio sobre la evolución de la página de Facebook Sentir Canario Gobcan.es durante su primer año de andadura son los siguientes:

- Conocer la dinámica de funcionamiento de esta red social: frecuencia de las publicaciones, horario y secciones.

- Determinar el tipo de contenidos publicados y diferenciar la producción propia de la externa.

- Saber qué tipo de fuentes se utilizan y establecer una clasificación en función de su origen.

- Concretar el nivel de participación de las entidades canarias del exterior en los contenidos de la página.

\section{Metodología}

Se planteó una investigación cualitativa con alcance exploratorio, y para ello, se utilizó la técnica de análisis de contenidos a través de las publicaciones del espacio Sentir Canario Gobcan.es desde el 3 de julio de 2017 al 2 de julio de 2018, es decir, un año natural. A través de dicho estudio se analizaron distintas variables como el número total de publicaciones, el horario en el que se efectuaban, la frecuencia, los contenidos y las fuentes. 
Además, se realizaron entrevistas con la persona encargada de la gestión de los contenidos de la página desde el mes de octubre de 2017 y con el Director General de Emigración del Gobierno de Canarias, José Manuel Téllez Ledesma, impulsor del proyecto.

\section{Análisis}

El total de post publicados durante el año que duró la investigación fue de 609. La norma general por lo que respecta a la publicación es la inserción de dos post cada día: uno por la mañana y otro por la tarde, salvo los sábados y los domingos, en los que solo hay uno en horario de tarde-noche. A lo largo del análisis se ha constatado que hay varios días sin publicación alguna, con la siguiente distribución por meses: enero (1), febrero (4), marzo (2), mayo (3) y junio (4) de 2018; en total son 14. Dado que las publicaciones tienen que tener el visto bueno del responsable político del proyecto, es decir del Director General de Emigración, los días en los que no ha habido publicaciones es porque no se ha dado dicha circunstancia.

El horario de publicación ha variado en tres ocasiones durante el año analizado. Empezó con la frecuencia siguiente: a las 11.00 horas de la mañana y a las 18.00 horas de la tarde; después a las 12.00 y a las 18.00 horas; y finalmente a las 13:45 y las 20:45 en los últimos meses del período estudiado. La elección de esta última franja, según la encargada de la gestión de los contenidos desde el mes de octubre de 2017 (anteriormente hubo otras dos personas), se debe a que, tras analizar los posibles horarios de emisión, se estimó que este último era el más pertinente y el que daba los mejores datos de audiencia. Hay que tener en cuenta que el público objetivo de esta página está constituido básicamente por los emigrantes canarios y sus descendientes que viven en varios países de América Latina con las correspondientes diferencias horarias, a lo que se añade los problemas de conexión a la red existentes en algunos de ellos.

En cuanto al tipo de contenidos desde el punto de vista de su producción, destaca el alto número de vídeos publicados, con un total de 141 , lo que supone el $23.15 \%$ del total. Los vídeos, tanto de producción propia como generados por organismos o particulares, son en su mayoría sobre el patrimonio natural de las islas (con el objetivo de ponerlo en valor y destacar la riqueza que en este aspecto tiene el Archipiélago); actuaciones musicales o bailes; y gastronomía típica de las islas. También hay vídeos especiales con motivo de las fiestas de Navidad, Año Nuevo y día de Reyes, así como por el día de Canarias con la participación de los emigrantes y descendientes. El mes en el que más vídeos se publicó fue junio (22), seguido de abril (21), ambos de 2018, y, por contra, en los que menos fueron septiembre y noviembre de 2017, con 6 cada uno. Y evidentemente, julio de 2018, con 1, ya

Facultad de Ciencias Sociales y de la Comunicación

Universidad de La Laguna

Avenida César Manrique, s/n; Campus de Guajara

38071 La Laguna, Tenerife (Islas Canarias - España) 
que en este caso solo se han tenido en cuenta dos días para el análisis. Hay una cantidad importante de vídeos insertados que tienen la marca del departamento de Promoción Turística del Gobierno canario.

Respecto a los contenidos provenientes de las entidades canarias en el exterior, o de canarios o descendientes, cabe reseñar que Sentir Canario Gobcan.es publica las noticias relacionadas con las actividades que desarrollan las distintas casas, centros y asociaciones canarias existentes fuera de las islas. La temática y los contenidos son tan variados como el tipo de actividades o acciones que llevan a cabo (fiestas, talleres, reuniones, participación en ferias y en competiciones deportivas, actos de homenaje, celebraciones religiosas, clases de bailes regionales, etc). Según la encargada de la gestión de los contenidos de la página, en las entidades no hay especialistas en comunicación, salvo en casos muy concretos como el Hogar Canario Venezolano Oficial de Caracas, por lo que las fotos, vídeos o contenidos son suministrados básicamente por las juntas directivas de dichas entidades con las que ha establecido contacto. Es decir, básicamente, los presidentes y secretarios de estas organizaciones.

Por otro lado, cabe destacar que, mientras que los contactos con Argentina y Venezuela son muy frecuentes, en el caso de Cuba hay muchas dificultades que se han ido subsanando con el paso de los meses. Estas circunstancias se plasman en la gran cantidad de post sobre las entidades de estos dos países frente a la escasez del resto.

Además de visibilizar las actividades que llevan a cabo dichas entidades, el objetivo del proyecto, según explica el Director General de Emigración, es dar a conocer los programas que ejecutan desde el área de Acción Exterior y disponer de una herramienta de consultas y resolución de problemas.

\subsection{Resultados}

\subsubsection{Contenidos externos}

Publicaciones de la emigración canaria en Argentina: 47, lo que supone un 7,71\% del total. Hay cuatro entidades canarias en Argentina (dos en Buenos Aires, una en La Plata y la otra en Rosario). Todos los meses hay post relativos a las actividades que desarrollan. En un total de tres meses hay hasta seis publicaciones, mientras que en cinco meses hay cuatro inserciones. Solamente en enero y febrero de 2018 hay una cada mes. En la práctica totalidad de los casos la fuente de la que provienen las fotos o vídeos son los centros existentes en dicho país. La mayoría de los post se refieren a celebraciones de distinto tipo, actividades musicales, homenajes y también destacan los talleres para personas mayores. 
Publicaciones sobre actividades relacionadas con Venezuela: Son un total de 92 las publicaciones relativas a actividades realizadas en Venezuela, lo que supone un 15,10\%. En este caso hay que señalar que 4 se refieren a la labor desarrollada por la delegación del Gobierno de Canarias en ese país vinculada a los programas que ejecuta el área de Acción Exterior, y 1 a una noticia sobre la realización de trámites en el Consulado de España en Caracas. Asimismo también varían notablemente las fuentes de las que proceden los contenidos de las publicaciones ya que en 9 ocasiones la fuente es el medio digital canariosenelmundo.com (1 en julio; 4 en agosto; y 4 en septiembre de 2017). Cabe destacar que el número de centros existentes en este país es de 57 . Hay publicaciones todos los meses salvo en noviembre de 2017. Por lo general, se trata de vídeos, fotos o de informaciones que proporcionan las entidades venezolanas que llevan a cabo todo tipo de actividades como las deportivas, folclóricas, festivas, de apoyo a los mayores, talleres, así como encuentros entre sus miembros, etc.

Publicaciones de la emigración en Uruguay y Paraguay: 13, lo que supone el 2.13\%. Son cuatro las entidades con sede en Uruguay. Hay publicaciones en septiembre y diciembre de 2017; y enero, febrero, marzo, abril y junio de 2018, destacando este último mes con cuatro entradas. Las entidades más representadas son ECICU Identidad Guanche y Descendientes Canarios Buscando Raíces de Tala. También tienen entrada la Asociación Islas Canarias de Maldonado y la Sociedad Islas Canarias de Montevideo. En este caso las publicaciones se refieren a aniversarios, fiestas, homenajes, figuras canarias ilustres emigradas a ese país, talleres y actividades musicales con niños. La única referencia a Paraguay es sobre la poetisa Josefina Pla.

Publicaciones de la emigración canaria en Estados Unidos: 13, un 2,13\% correspondientes a las comunidades de Los Isleños residentes en San Antonio de Texas y en Luisiana. Las publicaciones empiezan en diciembre de 2017 con la exposición del proyecto Cislanderus desarrollado por un grupo de investigadores para rescatar la memoria de los isleños en Estados Unidos. En 2018 la distribución es la siguiente: enero (2) fotos del museo de los isleños en Luisiana y de un isleño pionero de la música cajún; febrero (2): el cartel de la 43 Fiesta de Los Isleños y la portada de un libro sobre los mismos; marzo 2 vídeos: uno sobre la Fiesta de Los Isleños y otro sobre la celebración de los 300 años de historia de los canarios en San Antonio; abril (3): un vídeo sobre el monumento a las familias canarias que fundaron San Antonio; fotos en la celebración en honor al primer presidente norteamericano; y el cartel de la fiesta del rey William en San Antonio; y mayo (2): fotos en el desfile de la fiesta del Rey William y de la reunión para tener nuevos socios de la Asociación de Descendientes canarios de San Antonio. Finalmente, en junio de 2018 se inserta un vídeo sobre la actuación de la cantante canaria Fabiola Socas en Los Isleños Fiesta. La presencia

Facultad de Ciencias Sociales y de la Comunicación

Universidad de La Laguna

Avenida César Manrique, s/n; Campus de Guajara

38071 La Laguna, Tenerife (Islas Canarias - España) 
de los descendientes de canarios en el sur de Estados Unidos es muy notoria y desarrollan una gran actividad a la vez que cuentan con museos en los que se testimonia la presencia de la huella canaria desde el siglo XVIII.

Publicaciones de comunidades de Cuba, Puerto Rico y República Dominicana: La presencia isleña también se constata en estas islas del Caribe a través de las actividades desarrolladas por varias entidades cuya publicación se inicia a partir de diciembre de 2017. El número de post durante el período estudiado asciende a 13, un $2,13 \%$. La referidas a la isla de Puerto Rico (4) son sobre actuaciones de rondallas; celebración en el municipio de Hatillo en Puerto Rico; Festival Herencia Canaria en el Club de Amistad Canaria de Puerto Rico; y fotos de Domingo Bello, naturalista canario que emigró a esta isla.

Respecto a actividades realizadas en la isla de Cuba (7), se publica una infografía con imágenes y textos de emigrados a Cuba; fotos de escolares en un colegio cubano en el transcurso de una actividad relacionada con la emigración; imágenes del monumento al emigrante en Cuba; fotos sobre Leonor Pérez Cabrera; fotografías del Bar La Eminencia, referente de los canarios emigrados a esta isla; fotos del pescador lanzaroteño Gregorio Fuentes (amigo de Hemingway) que emigró a Cuba; y un vídeo con voces de la emigración canaria en la isla (proveniente de la Asociación de Mujeres).

También hay fotos de 1956, testimonio de la emigración canaria a República Dominicana y sobre la fundación de la provincia de Samaná.

Publicaciones de entidades existentes en el resto del territorio español: 46, lo que supone un 7,55\%. Son 8 las entidades canarias distribuidas por el territorio peninsular, ubicadas en Sevilla, Valencia, Murcia, Baleares, Madrid, Zaragoza, Valladolid y Barcelona. Algunas entidades son especialmente activas, como es el caso de las de Valencia, Baleares, Sevilla y Madrid. Mientras que otras, como las de Valladolid y Zaragoza, apenas tienen presencia en la página. Salvo en julio de 2017, todos los meses hay publicaciones acerca de sus actividades. El primer post insertado corresponde a la coronación de la reina de la Casa Canaria de Valencia en agosto de 2017.

El Hogar canario de Sevilla es el protagonista del Encuentro de Casas regionales que se celebra cada año en dicha ciudad andaluza. En octubre de 2017 tuvo lugar el IX Encuentro, dedicado a la comunidad canaria, y al mismo asistieron tanto el Viceconsejero de Acción Exterior, Luis Padilla, como el Director General de Emigración del ejecutivo regional.

Por su parte, el Hogar Canario en Baleares desarrolla actividades vinculadas a algunas festividades, como la del Pino o la fiesta de finados, y participa en eventos como la Feria del Tapeo, la Feria de la Constitución o la Diada de Baleares. Asimismo se imparten clases de 
baile regional o se organizan concursos de disfraces en los que destaca la participación de los más pequeños.

En cuanto a la Casa Canaria de Madrid, entre sus actividades cabe reseñar la Romería canaria que tuvo lugar el 9 de junio de 2018, el desarrollo de un encuentro literario con la colaboración de Canarias Crea, la puesta en marcha de una Escuela de Idiomas, y una velada para recaudar fondos para sufragar los gastos de la estatua en homenaje a los canarios en San Antonio de Texas.

A esto hay que añadir los post de la casa de Canarias en Berlín (6) así como del emigrante residente en México, Jorge Monterrey Hernández (3) y de la artista residente en Bélgica, Nayra Martín Reyes (1).

\subsubsection{Contenidos de producción propia (marca Sentir Canario):}

Los contenidos cuya producción corresponde a los gestores de la página se distribuyen en los siguientes conceptos:

-15 tarjetas de presentación de las casas canarias y/o entidades en el exterior: Hay una infografía insertada a los pocos días de iniciar su actividad la página, con un mapa en el que se sitúa el total de centros canarios distribuidos por continentes, según su ubicación en los distintos países. A partir de ahí, comienza la publicación, sin periodicidad fija, de las tarjetas de presentación de dichas entidades con una foto y los datos de identificación, localización, ubicación y teléfonos de las mismas. Cada tarjeta tiene el logotipo de Sentir Canario.

-Con la marca Sentir Canario hay varios vídeos especiales, como son los de felicitación con motivo de las fiestas de Navidad, Año Nuevo, día de Reyes, y el día de Canarias que cuentan con la participación de los emigrantes y descendientes. En mayo de 2018, además del vídeo del día 30, hay 4 vídeos denominados 'Me siento Canario' con los testimonios de los emigrantes que se publican antes del especial de felicitación del día de Canarias.

Por otra parte, en julio de 2017, cuando la página iniciaba su andadura, destaca un vídeo sobre una importante fiesta popular en las islas: el embarque de la Virgen del Carmen en Puerto de la Cruz (Tenerife).

-Fotos: Hay un apartado de fotos antiguas denominado 'Ayer y hoy' para el que se solicitó la colaboración de los seguidores de la página (aportando fotografías) en el que se insertan dos fotos de un mismo lugar (una antigua y otra actual) para ver si la audiencia identifica cuál es el sitio. La sección se mantiene en los meses de octubre y noviembre de 2017.

-Sorteos: En diciembre de 2017 se promueve el sorteo de una pintadera canaria y se publican varios anuncios haciendo un llamamiento a los seguidores para que participen 
contestando a una pregunta. El resultado del sorteo se publicó el 30 de diciembre de dicho año. En todo el período analizado es el único sorteo que se realizó.

-Fotos de perfil y fotos de portada: A lo largo del año estudiado se produjo en varias ocasiones el cambio de la foto de perfil y de la foto de portada. En concreto, cabe destacar el logotipo con el lazo negro con motivo de los atentados terroristas de Barcelona, publicado el 18 de agosto de 2017, y la actualización de la foto de portada el día 13 de diciembre con la tarjeta de felicitación de Navidad y Año Nuevo. El 9 de enero de 2018, una vez finalizadas las fiestas, se actualizan de nuevo tanto la foto de portada como la foto de perfil.

\section{-Infografías, fotografías y carteles:}

Algunos de los post publicados son infografías, fotografías y cartelería referida a distintos eventos:

-Foto de la Virgen de Candelaria y poema de felicitación, el 15 de agosto de 2017.

-Fotografías del 250 aniversario de la basílica de Teror, el 4 de septiembre de 2017.

-Cartel con fotos de felicitación por parte de Sentir Canario con motivo de la noche de finados, el 31 de octubre de 2017.

-Cartel dando las gracias por tener 1.000 seguidores, el 3 de noviembre de 2017.

-Tarjeta con fotos de las fiestas de la Virgen de la Candelaria en el mes de febrero de 2018.

- Programa de radio: Una de las particularidades de la página es que, a partir del lunes 14 de agosto de 2017, se inició una sección destinada a insertar un programa de radio semanal con el nombre de Sentir Canario radio, que, como regla general, se publica los viernes por la tarde. Salvo el correspondiente al viernes 22 de diciembre de 2017, que se pasa al domingo 24, día en el que se publica un especial de Navidad; y los viernes 16 de febrero y 16 de marzo de 2018 en los que tampoco hay programa. En el mes de mayo de 2018, además de los viernes, también hay un programa especial con motivo del día de Canarias el miércoles 30. Finalmente, en junio de 2018 tampoco hay los viernes 1 y 29 . El número de programas de radio realizados e insertados en el período estudiado asciende a 44 (7,22\% del total). Según manifiesta el Director General de Emigración, el programa de radio no estaba en el proyecto original sino que fue una iniciativa posterior que se impulsó por indicaciones de Presidencia del Gobierno. La producción y realización del mismo corresponde a la empresa Creación TV, dirigida por Francisco Almagro. Al frente del mismo hay dos locutores. Además de las entrevistas a canarios residentes en el exterior, incluye una sección para consultas sobre trámites relativos a la emigración, de la que se encarga la especialista Amaya 
Oyarzun. También hay una sección en la que se explica el significado distinto que tiene una misma palabra en varios países de habla hispana.

\subsubsection{Otros contenidos}

Durante los meses de julio, agosto, septiembre y octubre de 2017, los sábados por la tardenoche se insertaba un post con el programa emitido por la Televisión Canaria Noche de Taifas. En total son 16 las emisiones publicadas. Se trata de un programa de folclore, que se realiza rotatoriamente en los distintos municipios de las islas, en el que hay actuaciones musicales de grupos locales y de proyección regional.

La mayor parte de los contenidos de la página se refiere a actividades musicales de distinto tipo (95) y a fiestas (61), lo que supone el 25,61\% del total. Otros temas destacados son los vinculados al patrimonio natural de las islas (39) y el deporte (38).

\section{Fuentes}

El tipo de fuentes utilizadas para la elaboración de los contenidos de la página es muy variado. Además de las entidades y centros canarios ubicados fuera de las islas, las fuentes son básicamente institucionales, medios de comunicación y páginas de Facebook.

Por lo que respecta a las fuentes institucionales, destacan las siguientes: 1. Portal de noticias del Gobierno de Canarias, sobre todo en los tres primeros meses; 2. Página web del Instituto de Astrofísica de Canarias (IAC); 3. Página web de Ciencia Canaria, dependiente de la Agencia Canaria de Investigación, Innovación y Sociedad de la Información del ejecutivo canario; 4. Página web de Juventud Canaria, perteneciente a la Dirección General de Juventud del Gobierno autonómico; 5. Página web del Cabildo de La Gomera; 6. Página web de Promoción exterior (Proexca); 7. Página web del Boletín Oficial del Estado (BOE); 8. Página web de Promotur, web oficial de turismo de Canarias; 9. Página web del Ministerio de Exteriores español; 10. Página web del Instituto Tecnológico de Energías Renovables (Iter); y 11. Página web del Instituto Tecnológico de Canarias (ITC).

En cuanto a los medios de comunicación, se recurre básicamente a las ediciones digitales de las cabeceras de referencia o a medios nativos digitales.

Ediciones digitales de periódicos de cabecera, revistas y agencias de noticias: 1. www.laopinion.es; 2 www.canarias7.es; 3. www.diariodeavisoselespañol.com; 4. www.eldia.es; 5. ABC.es; 6. www.elviajero.elpais.com; 7. www.laprovincia.es; 8. www.nationalgeographic.com.es; y 9. Efe Verde. 
Periódicos nativos digitales: 1. www.canariosenelmundo.com, con 15 entradas; 2. www.lagomera.es; $3 . \quad$ www.canariasenhora.com; $4 . \quad$ www.diarioelhierro.es; 5. www.teldeactualidad.com; y 6 . www.diariodetenerife.info.

Programas de televisión: 1. http://www.rtvc.es/television/programa/noche-de-taifas12233.aspx; y 2. http://www.rtve.es/alacarta/videos/aqui-la-tierra/.

Asimismo hay un importante número de fuentes digitales de variada temática y procedencia, ya que hay páginas de turismo, gastronomía, cultura, música y entidades. Dichas referencias son, entre otras: 1 . //mailchi.mp; 2. noticias.universia.es; 3 . Wikipedia; 4 . www.salyroca.es; 5. http://canaryislands.demola.net; 7. https://ilovetheworld.es; 8. www.canaryfly.es; 9. www.obrasociallacaixa.org; $10 . \quad$ www.cislanderus.com; 11. https://saboreandocanarias.com/2018/10/18/tenerife-gastro-experience-desde-el-productohasta-la-cocina/; 12. www.losggofiones.com; $13 . \quad$ www.visitlapalma.es; 14. www.cuevadelviento.net; 15. www.cida-sa.org; y 16. wwww.enjoyfuerteventura.net.

Finalmente hay que destacar la utilización de páginas de Facebook de entidades, instituciones y organismos, así como de artistas.

En el uso de las fuentes se observa la presencia bastante significativa de fuentes de organismos y entidades oficiales tanto locales como regionales y estatales así como de medios de comunicación. Sin embargo, hay fuentes como Wikipedia que no ofrecen mucha credibilidad.

En algunos casos, en torno al $9 \%$ del total de los post publicados, no se ha podido determinar cuál es la fuente de la información que se inserta dado que se trata de post en los que los textos son de producción propia pero no hay referencia a la procedencia de las fotos o los vídeos que los acompañan.

\section{Conclusiones}

Canarias es la única comunidad autónoma española que cuenta con una página de Facebook concebida como canal de comunicación y de visibilización de las actividades que desarrollan los emigrantes y sus descendientes en el exterior con participación directa de los mismos. El País Vasco dispone de una red social con la misma filosofía pero se limita a replicar las publicaciones de las entidades vascas añadiéndoles un texto de referencia.

El funcionamiento de la página durante los primeros cuatro meses es un tanto errático: cambios en el horario de las publicaciones, secciones que aparecen y desaparecen, contenidos poco ajustados al público objetivo. Esto se explica en parte porque de julio a 
octubre hubo tres personas encargadas de la gestión de los contenidos. La situación se revierte a partir de noviembre de 2017.

La página presenta un índice significativo de post que son de producción propia en los que se plasma la marca Sentir Canario que se va consolidando como elemento identificativo de la comunidad canaria en el exterior y que está presente en las actividades que desarrolla (básicamente a través de la cartelería y los roll up) como se puede observar en las fotografías que remiten las entidades.

Durante su primer año, en torno al 35\% de las publicaciones de esta red social corresponden a las actividades que desarrolla la comunidad de emigrantes y descendientes que viven fuera de las Islas, destacando los casos de Venezuela, Argentina y los residentes en el resto del territorio nacional.

Hay un número importante de vídeos y de fotografías, primando el elemento audiovisual claramente en los contenidos. Los vídeos son tanto de producción propia como provenientes de fuentes oficiales y de las propias entidades o particulares que han emigrado. Son significativos los videos especiales producidos con motivo de las festividades más señaladas del año en los que se da voz a las comunidades en el exterior.

La utilización de fuentes de distinto tipo es considerable, sin embargo, en algunos casos dichas fuentes tienen escasa calidad, como es el caso de wikipedia. Además, hay post en los que no se puede determinar cuál es la fuente de la que proceden las fotos o los vídeos que se insertan.

\section{Bibliografía}

Consejo general de la Ciudadanía española en el exterior. Real Decreto 230/2008, de 15 de febrero, 8734 - 8737. Recuperado de https://www.boe.es/boe/dias/2008/02/16/pdfs/A0873408737.pdf

Consejos de residentes españoles en el extranjero. Real Decreto 1960/2009, de 18 de diciembre, 137 - 141. https://www.boe.es/boe/dias/2010/01/02/pdfs/BOE-A-2010-44.pdf

Dirección General de Emigración. Gobierno del Principado de Asturias. (20 de mayo de 2018). Noticias destacadas. www.emigrastur.es

Entidades Canarias en el Exterior y del Consejo Canario de Entidades en el Exterior Ley 4/1986, de 25 de junio, 30473 - 30474. https://www.boe.es/eli/escn/l/1986/06/25/4/dof/spa/pdf 
Estatuto de la ciudadanía española en el exterior. Ley 40/2006, de 14 de diciembre, 1-20. Recuperado de https://www.boe.es/buscar/pdf/2006/BOE-A-2006-21991-consolidado.pdf Generalitat de Catalunya (13 de mayo de 2018). Catexterior. www.facebook.com/Catexterior/ Grup Comunicació 21 (18 de junio de 2018). Comunicació21. El portal catalán del sector de la comunicación. https://comunicacio21.cat/

Grup Comunicació 21 (20 de julio de 2018). Exterior. El portal que explica que hace Cataluña en el mundo. https://exterior.cat/

Secretaría Xeral da Emigración da Xunta de Galicia (12 de mayo de 2018). Galicia Aberta. www.facebook.com/GaliciaAberta/

\section{Forma de citar este artículo en bibliografías}

ÁLVAREZ-DÍAZ, A. (2019): Gestión de RR.PP. Internacionales: la aventura de Sentir Canario Gobcan.es, en Revista PANGEA № 10, páginas 1 a 13. Tenerife: Red Académica Iberoamericana de Comunicación. Recuperado el _ de de 2 de: http://www.revistapangea.org 